\title{
Online conversation application with confidentiality, anonymity, and identity requirements
}

\author{
Pedro Fernandes ${ }^{1}$ and António Pinto ${ }^{2}$ \\ ${ }^{1}$ GCC, CIICESI, ESTG, Politécnico do Porto, Portugal \\ 8080084 eestg.ipp.pt \\ 2 GCC, CIICESI, ESTG, Politécnico do Porto \\ and CRACS \& INESC TEC, Porto, Portugal \\ apintodinesctec.pt
}

\begin{abstract}
The increase in usage of smartphones and the ubiquity of Internet access have made mobile communications services very attractive to users. Messaging services are among the most popular services on the Internet. In recent years, this services started to support confidentiality and anonymity. A recurrent problem with the existing messaging solutions is their lack of resistance to impersonation attacks. The proposed solution addresses the impersonation problem, without neglecting user confidentiality and anonymity, by forcing users to exchange the required cryptographic material among themselves. Moreover, this exchange must use a proximity communication technology, forcing the users to physically meet.
\end{abstract}

Keywords: Impersonation; Anonymity; Online conversation.

\section{Introduction}

The increase in usage of smartphones and the ubiquity of Internet access have made mobile communications services very attractive to users. Messaging services being among the most popular because these of its availability, functionality and lower costs of communication. In particular, these services make international communications free, if the user already has Internet connectivity, and very attractive due to functionalities as the use of emoji or photo and video sharing.

In recent years, these messaging services started to support end-to-end (E2E) encryption in order to protect the transmitted content from eavesdropping when used over unsafe communication channels. In E2E encryption, the messages are encrypted at the source terminal, sent through the network, and decrypted only at the destination terminal. Servers, if used, are expected to not be able to access the exchanged messages in clear text form [6]. This may not the be case if the server has access to the cryptographic material used to encrypt the messages. Whenever the server securely transfers messages from source to destination and is unable to access the messages, or to identify the user who sent the message, we 
are in presence of a secure messaging platform that enables both confidentiality and anonymity This is sometimes referred to zero-knowledge applications [8].

The interest in secure forms of sending online messages has grown substantially and lead the Electronic Frontier Foundation (EFF) to evaluate [3] the existing ecosystem of smartphone applications that offer secure messaging services. The list of messaging applications is very long and difficult to maintain due to the frequent appearance of new ones. This study is currently identified by EFF as outdated and motivated the authors to extend it.

All modern messaging applications, to the best of our knowledge, enable users to directly communicate without requiring them to confirm their real identify. This opens the possibility of a user assuming the identify of another, i.e. user impersonation. This is a problem that potentiates ill-intentioned users, possibly with criminal intent, to try and deceive more susceptible users such as children or less tech-savvy older people. The proposed solution, while maintaining users' privacy and anonymity, enables secure E2E communications, solves this user impersonation problem by requiring a first, physical interaction between the communicating users. This first interaction is based on a proximity communication technology.

The paper is organized in sections. Section 2 describes the related work, in particular it compares secure messaging applications. Section 3 details the proposed solution, which is then evaluated in Section 4. Section 5 concludes this paper.

\section{Related work}

A set of secure messaging applications was selected from the ones available in Google Play and App Store. The selection was based on the ones that better performed in the previously mentioned study of the EFF and that had the more installations. In particular, TextSecure, Signal, Telegram, WhatsApp, Threema and Wickr were selected.

TextSecure is a free and open-source mobile application for the Android platform that allows the to send encrypted text messages. It was first released in 2010 by Open Whisper Systems [12]. In October 2015, TextSecure had been installed over 1 million times through Google Play [9]. The protocol initially used by TextSecure was a protocol derived from the Off-the-Record (OTR) protocol [11]. It comprised four stages: 1) registering; 2) sending/receiving a first message; 3) sending of a follow-up message; 4) sending of a response. The application relays its messages to the destination to the server that, in turn, transmits the messages to the destination. The parties communicate with the server via Representational State Transfer (REST)-Application Programming Interface (API) over secure Hypertext Transfer Protocol (HTTPS). The delivery of the actual message is performed via Google Cloud Messaging (GCM) which basically acts as a message delivery intermediary [18]. Currently, TextSecure implements the algorithm Double Ratchet (also known as Axolotl ratchet). 
Signal is the successor of both the RedPhone (voice calls encryption) and of the TextSecure. Signal was launched in 2015 and is a secure instant messaging and voice call application that uses E2E encryption. The encryption keys of the users are generated and stored in their smartphones and not on the application servers [3]. Signal was built with mechanisms to resist Man-in-The-Middle (MiTM) attacks. For voice calls, the application displays a word on the screen, if the two words match at both ends of the call, then the call is considered secure $[13,15]$. There is a similar mechanism for messages that consists on the mutual verification of digital signatures. The protocol used by the Signal application is open source and is known as the Axolotl protocol.

Telegram provides E2E message encryption and self-destructing messages. Telegram has more than 100 million monthly active users. The application offers two types of chats: the standard chat that uses an encryption key that is shared with the server and can be accessed from multiple smartphones; and the secret chat (Telegram (Secret Chats)) that uses E2E encryption and can only be accessed by the devices that are in possession of the required cryptographic material. The adopted protocol, named MTProto, uses RSA2048 [1], Advanced Encryption Standard (AES) 256 bits and the Diffie-Hellman key exchange [2]. The MTProto protocol comprises three components [14]: the high-level component, the authorization component, and the transport component. The high-level component defines the method by which the API queries and responses are converted into binary messages. The authorization component defines the methods used for user authentication and messages encryption. The transport component defines the method to be used by clients and server for message transmission over a network protocol. The MTProto supports multiple transport modes, such as Hypertext Transfer Protocol (HTTP), HTTPS, Transmission Control Protocol (TCP) and User Datagram Protocol (UDP).

WhatsApp is another Internet messaging application. Numbers of September of 2015 put their user base in the 900 million users [10]. WhatsApp uses a store and forward approach for message transmission. When a user wants to send a new message, it is first stored on the WhatsApp server, and then the server relays the message to the destination user. Once the message is received by the destination, it is removed from the server database [5]. WhatsApp uses the same protocol as TextSecure (Axolotl or Double Ratchet algorithm).

Threema is another free and open source Internet messaging application with support for E2E encryption and user anonymity. As of June 2015, Threema had 3.5 million users, most of them from German-speaking countries. Each user, at the application start, receives a randomly assigned a Three-ID that will be used for user identification. In this process, neither the user mobile phone number or email address is required. Users can verify the identity of their Threema contacts by scanning their QR code when they physically meet. Using this feature, users can be sure that they contain the correct public key of their contacts, providing message confidentiality and resistance to MiTM attacks [16]. Threema was designed to store as little data on servers as possible. The contact lists are managed only on the users smartphones and messages are deleted 
immediately after they have been delivered. Threema supports E2E encryption [17].

Wickr is another free Internet messaging application that offers confidentiality. It includes the ability to set a time-to-live for each message. The recipient's application erases the encrypted message from their smartphones, trying to ensure that the message can no longer be retrieved. The Wickr Secure Messaging protocol is specifically designed to prevent servers from accessing both the keys or detailed information from users. Supports E2E encryption by implementing multiple encryption layers [7]. The data, stored or in transit, is encrypted with AES256. Each message is encrypted with a new encryption key, deleted after its use (Perfect Forward Secrecy). Message encryption keys are then encrypted with the public key (Elliptic Curve Diffie Hellman (ECDH) 521) of the recipient. All user content is deleted after the user logs off. The unique identifier of the smartphone, Unique Device Identifier (UDID), is never sent to the Wickr servers in an effort to assure user anonymity. Wickr's Secure Shredder erases all data on the smartphone so it can not be recovered.

Transport Provider Entities Anonymity Identity

\begin{tabular}{lccccc}
\hline Text-Secure & Yes & Yes & Yes & No & No \\
\hline Signal & Yes & Yes & Yes & Yes & No \\
\hline Telegram & Yes & No & No & No & No \\
\hline Telegram & & & & & \\
(Secret-Chats) & Yes & Yes & Yes & No & No \\
\hline WhatsApp & Yes & No & No & No & No \\
\hline Threema & Yes & Yes & Yes & Yes & No \\
\hline Wickr & Yes & Yes & Yes & Yes & No \\
\hline
\end{tabular}

Table 1. Application comparison

These applications were compared in the Secure Messaging Scorecard of EFF [4]. Table 1 extends this comparison for the selected applications. The comparison criteria is fivefold: 1) transport; 2) provider; 3) entities; 4) anonymity; 5) identity. If all communications are encrypted while being transported through the network, then the first criteria is satisfied. The second criteria requires that all communications must be encrypted E2E, which means that the keys needed to encrypt/decrypt messages must be generated and stored on the smartphones and not on the servers. The third criteria requires that there is an internal method for the verification of the identity of the involved entities and of the integrity of the channel, even when the server or third parties are compromised. The forth criteria requires that the identity of the user is not known by the server. The last criteria requires that the users can be sure of the other users identity because that had a physical interaction as proof of their identity.

For instance, the TextSecure application despite satisfying the three initial criteria, does not provide user anonymity nor requires any prior physical interaction between users in order to establish a conversation 
between them. Off the selected applications, only Threema permits the use of an interaction between the users as a form identity confirmation, but because it can be made remotely, undermines its fulfilling of the last criteria.

\section{$3 \quad$ Eko}

The architecture of the proposed solution, named Eko, is depicted in Figure 1. It comprises end user terminals, which are smartphones, and a server. An user X, in order to send a secure message to an user Y, prior to its upload to the server, firstly encrypts the message with the chat symmetric key $\left(S K_{x}\right)$, and then encrypts this result with a second chat symmetric key $\left(S S K_{x}\right)$. The $S K_{x}$ is only known by the users $\mathrm{X}$ and Y, while the $S S K_{x}$ is known be the users and the server.

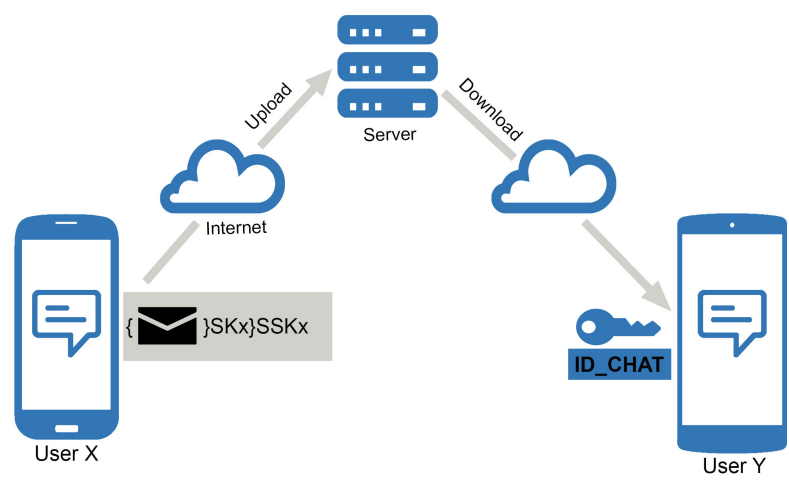

Fig. 1. Application architecture

All cryptographic material is generated at the smartphones, except for the $S S K_{x}$ that is generated by the server. The conversation identifier ( $\left.I d\right)$ is also generated by the server to avoid $I d$ collision. Each conversation is represented by a data structure named ID_CHAT that is detailed in Table 2 . In addition to $I d$ and the cryptographic material, it includes a time stamp and a message validity in number of days.

\begin{tabular}{ll}
\hline Variable & Description \\
\hline$S K_{i}$ & Conversation user key \\
$S S K_{i}$ & Conversation key shared between users and server \\
$I d$ & Conversation identifier \\
$T_{s}$ & Timestamp \\
$V$ & Validity $(\mathbf{1}, \mathbf{1 0}, \mathbf{1 5}, \mathbf{3 0}$ days $)$ \\
\hline
\end{tabular}


All data transmitted by the application uses E2E encryption, guaranteeing that all communications with other users is confidential. The server uses the conversation identifiers to select the appropriate decryption key to process each message but is unable to decrypt the message content and to obtain the identification of the users that take part in each conversation, thus providing anonymity.
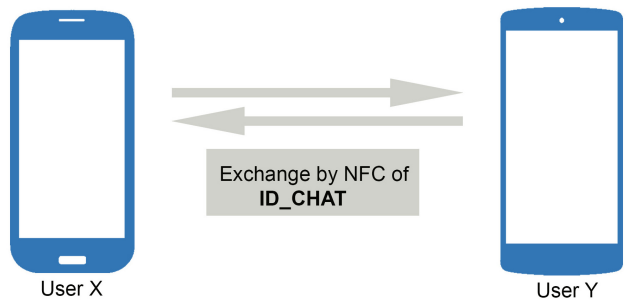

Fig. 2. $I D_{-} C H A T$ exchange between users

Users, in order to start exchanging messages with each other, first have to undergo a physical interaction consisting of exchanging an instance of the data structure $I D_{-} C H A T$ be means of a proximity communication technology. Example technologies being Near Field Communications (NFC) or Bluetooth Low Energy (BLE). The ID_CHAT data structure is generated by one user, and then directly shared with the remaining users, without interaction with the server, as shown in Figure2.

\section{Validation}

Prototype client and server applications were implemented to validate the proposed solution. The server was developed using the Laravel framework and contains the necessary access points for the operation of the clients. The client application was developed using the Ionic framework that allows multi-platform development. The functional assessment was successfully performed and the identified requirements were satisfied.

A security analysis was also performed considering the confidentiality, anonymity and impersonation. The confidentiality of the messages exchanged between client and server is obtained by means of E2E encryption. The keys used to encrypt messages in conversations, the $S K_{x}$ key, is generated in the smartphone of the user that creates the conversation and stored locally. It is never uploaded to the server. The second key, the $S S K_{x}$ key, is known by the participants of a conversation and by the server. Finally, being a web-based service, the server is deployed only in its secure mode (HTTPS).

The proposed solution guarantees user anonymity by the way it creates and uses the ID_CHAT data structure. The identity of the user that sends each messages is unknown to the server. Each message assumes the form:(chatId :Ts: $\left.\left\{\{U \text { sername }: M s g\}_{S K_{X}}: \text { chatId:Ts }\right\}_{S S K_{X}}\right)$, 
where $\{a\}_{b}$ means $a$ encrypted with key $b$, and $a: b$ mean the concatenation of $a$ with $b$. The identity of the user that sends each message can only be decrypted with the $S K_{x}$ key that exists only in the ID_CHAT data structure on the smartphones.

The proposed solution addresses the impersonation problem by imposing a previous interaction between users for the conversation to take place. This interaction comprises the generation of a new ID_CHAT data structure and its direct exchange with the other user's smartphone by means of a proximity communication technology. This way, the users must physically meet in order to communicate.

\section{Conclusion}

Messaging services are among the most popular services on the Internet. In recent years, this services started to support confidentiality and anonymity. A recurrent problem with the existing messaging solutions is their lack of resistance to impersonation attacks. The proposed solution addresses the impersonation problem without neglecting user confidentiality and anonymity. A prototype of the proposed solution was implemented and functionally verified. A analysis of the security of the proposed solution was also performed.

\section{References}

[1] Calderbank, Michael: The RSA Cryptosystem: History, Algorithm, Primes (2007)

[2] Demircioglu, Murat and Taskın, Halil Kemal and Sarımurat, Salim: Security analysis of the encrypted mobile communication applications (2014)

[3] Electronic Frontier Foundation: Secure Messaging Scorecard. Which apps and tools actually keep your messages safe?, last accessed 11 September 2016

[4] Electronic Frontier Foundation, Julia Angwin, Joseph Bonneau: Secure Messaging Scorecard. https://www.eff.org/secure-messagingscorecard (2014)

[5] Gaurav Rathee: How WhatsApp Works. http://digitalperiod.com/explore-whatsapp-clock-sign-and-tick/ (2015)

[6] Greenberg, A.: Hacker Lexicon: What Is End-to-End Encryption?, WIRED (2015), last accessed 17 March 2016

[7] Inc, W.: How Wickr's Encryption Works. https://www.wickr.com/security/how-it-works, last accessed 17 February 2016

[8] Pedersen, Cam and Dahl, David: Crypton : Zero-Knowledge Application Framework (2014), last accessed 2 March 2016

[9] Play, G.: TextSecure Private Messenger, last accessed 17 February 2016 
[10] Sun, L.: Facebook Inc.'s WhatsApp Hits 900 Million Users: What Now? The Motley Fool. http://www.fool.com/investing/general/2015/09/11/facebookincs-whatsapp-hits-900-million-users-what.aspx (2015)

[11] Systems, O.W.: Advanced cryptographic ratcheting. https://whispersystems.org/blog/advanced-ratcheting/, last accessed 28 September 2016

[12] Tactical Technology Collective and Front Line Defenders: TEXTSECURE FOR ANDROID, security in-a-box. https://securityinabox.org/en/guide/textsecure/android (2009)

[13] TechCrunch. AOL: Talk Private To Me: Free, Worldwide, Encrypted Voice Calls With Signal For iPhone, last accessed 13 September 2016

[14] Telegram: Mtproto mobile protocol

[15] The Zfone Project: Exactly how does Zfone and ZRTP protect against a man-in-the-middle (MiTM) attack?, last accessed 13 September 2016

[16] Threema GmbH: Threema

[17] Threema GmbH: Threema Cryptography Whitepaper. https://threema.ch/en/faq, last accessed 10 September 2016

[18] Tilman Frosch, Christian Mainka, Christoph Bader, Florian Bergsma, Jorg Schwenk, Thorsten Holz: How Secure is TextSecure? (2014) 